Gerhard Boysen*

\title{
Comparaison et reversibilité en français
}

\section{Introduction}

La catégorie de la comparaison n'est pas traitée d'une manière très fouillée dans les grammaires du français. Un travail comme celui d'Eric Buyssens ${ }^{1}$, sur les catégories grammaticales, ne mentionne même pas la comparaison. Quand René Rivara commence son ouvrage "Le système de la comparaison" 2 par ces termes, auxquels nous souscrivons entièrement:

"Si l'on consulte les grammaires les plus connues de quelques grandes langues indo-européennes, français, latin, anglais, allemand, italien, on ne peut qu'être frappé par le peu d'intérêt que semble avoir suscité le phénomène, syntaxique et sémantique, de la comparaison” (p. 15),

cet auteur n'échappe pas tout à fait à la même critique: dans son ouvrage, il ne souffle mot, en tout cas, du problème qui sera le sujet de cet article: l'opposition comparaison analytique et comparaison synthétique. Pourtant, les grands maîtres de la linguistique n'ont pas jugé indigne de s'occuper de la comparaison: je pense à Louis Hjelmslev ${ }^{3}$ et à Emile Benveniste ${ }^{4}$.

Si nous prenons un exemple comme

(1) Paul est plus grand que Pierre,

nous pouvons sans difficulté transposer l'adjectif au positif:

(1') Paul est grand.

1 Les catégories grammaticales du français, Bruxelles 1975.

2 Paris 1990.

3 Sprogsystem og sprogforandring, København 1972, p. 61-90.

4 Comparatifs et superlatifs, dans: Noms d'agent et noms d'action en indo-européen, Paris 1975 (1948), p. 114-68.

* Gerhard Boysen

Institut de langue et de communication

Université d'Odense

Campusvej 55

DK-5230 Odense $M$ 
Par contre, avec un exemple comme

(2) Il s'est mis à se frapper la poitrine, à dire qu'effectivement comme salaud on ne faisait pas pire que lui (Jean Hougron, Histoire de Georges Guersant 363)

il n'est pas possible de remplacer pire par mauvais:

*on ne faisait pas mauvais

Je propose de rendre compte de cette différence en employant le terme de réversibilité.

Un comparatif ou un superlatif est réversible s'il peut être remplacé par le positif du même adjectif dans le même contexte, sans autre altération du sens que celle du sens comparatif ou superlatif.

Prenons un autre exemple:

(3) Il ne pouvait plus se passer de drogue. C'était pire que d'avoir envie de faire l'amour trois fois par jour (Thérèse de Saint Phalle, Le Souverain 202).

Dans cet exemple, on peut sans difficulté transposer le superlatif pire à un adjectif au positif; seulement, cet adjectif ne sera pas mauvais:

(3') Il ne pouvait plus se passer de drogue. C'était aussi mauvais que d'avoir envie de faire l'amour trois fois par jour

serait possible, mais aurait un autre sens (mauvais aurait plutôt le sens de 'nuisible', 'mauvais pour la santé'). Pour obtenir le même sens que dans (3), il faudrait employer un adjectif comme dur:

(3") Il ne pouvait plus se passer de drogue. C'était aussi dur que d'avoir envie de faire l'amour trois fois par jour.

Ceci semble indiquer qu'il faut distinguer plusieurs types de réversibilité:

- réversibilité morphologique, qui permet de transposer un comparatif/superlatif à un adjectif au positif;

- réversibilité lexicale, qui permet de transposer un comparatif/ superlatif au positif du même adjectif.

La réversibilité lexicale présuppose la réversibilité morphologique.

Dans (1), nous avons une réversibilité lexicale, dans (3) une réversibilité morphologique, tandis que (2) présente une non-réversibilité totale. 
Le terme de réversibilité a été employé dans la grammaire transformationnelle pour indiquer le caractère particulier de certaines transformations ${ }^{5}$, mais, à part cela, il n'a pas, que je sache, été beaucoup utilisé comme terme technique en linguistique.

Pour introduire un nouveau terme, il faut des arguments convaincants et sérieux. Il me semble, en effet, qu'il y en a. Un des avantages de ce terme est qu'il rend superflus les termes de "comparatifs absolus" et "superlatifs absolus", qui ont représenté des croix, pour beaucoup de linguistes (p. ex. P. Tekavc\&id, Grammatica storica dell'italiano § 487).

Mais il y a aussi des avantages plus importants. Une distinction entre comparatifs réversibles et comparatifs irréversibles rendra compte, dans une grande mesure, d'un problème qui n'a jamais été résolu: la concurrence entre formes comparatives analytiques et synthétiques.

Non seulement beaucoup de grammaires réservent à cette question un traitement fort cavalier, mais la description qu'on trouve dans les grammaires - même dans des grammaires réputées solides et consciencieuses - sont souvent inexactes:

"Pire (...) est moins courant que plus mauvais". (Grevisse-Goosse $\S 554)$

"Pire est en concurrence avec plus mauvais. Ce dernier tend à l'emporter aujourd'hui" (W. von Wartburg og P. Zumthor, Précis de syntaxe du français contemporain §528)

"Pire et pis (...) sont en concurrence avec plus mauvais et plus mal; on les empoie surtout dans des expressions figées: Le remède est pire que le mal". (Grammaire Larousse §304).

"Pire et pis sont bien moins employés que plus mauvais et plus mal dans la langue courante; pire tend même à empiéter sur pis qui ne subsiste plus guère que dans des locutions figées" (Gougenheim, Système grammatical de la langue française, p. 255-56).

"Tre adjektiver har en syntetisk form i komparativ, heraf har de to [mauvais, petit] en hyppigere anvendt analytisk form ved siden af (...). (Pedersen, Spang-Hanssen, Vikner: Fransk Grammatik §47.2).

"Pire et pis. Ces deux comparatifs sont littéraires et d'un usage peu étendu. On leur préfère en général plus mauvais et plus mal" (Knud Togeby, Grammaire française I, §250).

5 voir Jean Dubois e.a., Dictionnaire de linguistique et des sciences du langage, Paris 1994 (réversible). 
Or, ces assertions sont tout simplement fausses. Pire n'est pas moins fréquent que plus mauvais: les chiffres de mon corpus, qui représente environ 43.000 pages de la période 1960-76, montrent une répartition de moins de $10 \%$ d'emplois de plus mauvais et de plus de $90 \%$ de pire. Mon propos dans cet article est de montrer que cette différence peut, en grande partie, être ramenée à la distinction comparatif réversible/irréversible.

Si je tiens à cette notion de réversibilité, c'est qu'elle couvre les deux aspects fondamentaux de la distinction comparaison synthétique/ analytique: l'aspect scalaire (l'aspect de graduation) et l'aspect lexical. Le terme de scalaire ne rendrait compte que du premier de ces deux aspects. Ainsi, on peut très bien avoir un comparatif scalaire, qui n'est pas lexicalement réversible (type de pires ennemis $=$ de plus grands ennemis).

Je propose de formuler une loi générale disant qu'un comparatif ou superlatif analytique est toujours réversible. Un comparatif ou un superlatif synthétique est le plus souvent non-réversible, mais peut être réversible.

Je procéderai en examinant d'abord le comparatif, ensuite le superlatif des deux adjectifs qui offrent, en français moderne, une alternance forme analytique - forme synthétique, mauvais et petit.

\section{Comparatif}

\section{1. pire - plus mauvais}

Tous les 26 exemples analytiques de mon corpus sont des exemples réversibles:

(4) La situation était encore plus mauvaise qu'au départ (Hubert Monteilhet, Le retour des cendres 158).

Par contre, la grande majorité des 285 exemples avec la forme synthétique sont du type non-réversible; dans la plupart des cas lexicalement non-réversible.

On peut distinguer, à l'intérieur des cas de non-réversibilité, plusieurs types:

- le type où pire fonctionne comme un intensif de l'adjectif, où il pourrait être remplacé, au positif, non pas par mauvais, mais plutôt par grand: 
(5) C'est un fléau pire que la lèpre (Pierre Boulle, Les oreilles de la jungle 128).

C'est un emploi de pire que l'on rencontre avec des substantifs comme catastrophe, désastre, souffrance, supplice, tyrannie, ou, pour prendre aussi un exemple avec un substantif animé:

(6) ces "enquêteurs" (...) qui, pour moi, ne peuvent être de pires ennemis que vous (Guy des Cars, La vipère 278).

Dans ces exemples, le comparatif fonctionne donc comme un intensif de l'adjectif. Parfois, l'intensité est tellement forte que le comparatif pourrait être remplacé par plus:

(7) C'était quelque chose de pire que la misère: la gêne, l'étroitesse, la minceur (Georges Pérec, Les Choses 87)

- dans d'autres cas, le comparatif ne marque pas une intensité ("grand"), mais correspond plutôt à grave:

(8) - Tu as une crise de sciatique? Je suis sûre que tu as pris froid... -Non, ce n'est pas la sciatique (...). C'est bien pire que la sciatique. (Jean-Louis Curtis, Le thé sous les cyprès 201)

ou dur:

(9) La liberté, vingt dieux! Ils nous ont assez empoisonnés avec la liberté! Eh bien, elle n'est pas à sens unique, la liberté! Le contrôle économique français était pire que les Allemands! (Armand Lanoux, Quand la mer se retire 164).

Dans ces exemples, pire n'est pas lexicalement réversible: si on transposait les adjectifs au positif, ce n'est pas mauvais qu'on trouverait, mais plutôt grave et dur.

- Parfois, le contexte nous fournit l'adjectif, la "base lexicale" sur laquelle est construit le comparatif pire:

(10) Bien que cet accrochage ait été très pénible et nous ait tous remplis de honte, le silence qui suit est mille fois pire (Robert Merle, Madrapour 260):

pire $=$ plus pénible.

Les cas où pire est non-réversible morphologiquement sont surtout des cas où pire apparaît seul. En plus de l'emploi au sens neutre, que nous avons vu dans (2) (faire pire, etc.), il s'agit des emplois suivants:

après préposition: en pire:

(11) Et tout avait recommencé comme l'année précédente, en pire (Roger Ikor, Le Tourniquet des innocents 261) 
et à pire:

(12) -Attends-toi à pire que ce que tu as connu (Henri Charrière, Banco 586);

en apposition:

(13) Ainsi ne risquait-il pas le clin d'oeil complice ou, pire, engageant qu'Isabelle aurait pu surprendre. (Luc Estang, La fille à l'oursin 124)

ou adverbialement

(14) Il banquillait à toute allure, il cahotait pire qu'un canard très pressé (Hougron, Guersant 439).

Tous ces cas de non-réversibilité de pire écartés, la question reste alors: pour les exemples qui demeurent - les exemples de pire réversible quelle est la différence avec plus mauvais?

Les cas de non-réversibilité écartés, nous sommes en présence d'une distribution beaucoup plus harmonieuse des deux formes, non seulement numériquement (26 ex. - 50 ex.), mais aussi du point de vue qualitatif. Il est frappant que les exemples de plus mauvais et les exemples de pire se rencontrent dans des contextes - avec des substantifs noyaux - dans une grande mesure identiques, ce qui confirme que l'opération d'écarter les emplois non-réversible a été pertinente. Ce sont des substantifs neutres comme condition, destin, position, situation, vie:

(15) Au fond, ma position n'est pas plus mauvaise qu'à Paris (Françoise Mallet-Joris, Les signes et les prodiges 340)

(16) et à la rentrée la situation redeviendra la même, ou pire (id., Allegra 329)

et des substantifs comme résultat, ou des types de résultats ou de produits (récolte, peinture, roman):

(17) et comme Van rangeait escabeau et outils, elle reconnut que le résultat aurait pu être plus mauvais (Pierre Boulle, Les oreilles de la jungle 187)

(18) La fabrication en série, qui allait commencer, donnerait sans doute des résultats bien pires (id., Le jardin de Kanashima 60).

Devant de tels exemples, je ne vois qu'une explication, c'est d'attribuer à la forme analytique une valeur expressive (emphatique), qui n'est pas dans la forme synthétique. 
Quand il s'agit d'un substantif humain, on emploie en général la forme synthétique:

(19) Plus rien ne m'accroche. Il n'y a pas pire lecteur que moi (Simone de Beauvoir, La femme rompue 67)

(20) Tavernier n'était pas pire que les autres et, près de certains ivrognes de l'immeuble, il faisait même figure de bon père de famille (Jean Hougron, Les Humiliés 168)

tandis que la forme analytique s'emploie, rarement, avec une valeur emphatique:

(21) Tu verras c'est pas une déesse, mais au lit y en a des plus mauvaises (Bertrand Blier, Les Valseuses 346)

(22) Il dit au dos de Thérèse qu'elle est plus mauvaise qu'une gale (Suzanne Prou, La terrasse des Bernadini 75).

Dans (22), il s'agit d'un emploi particulier, où mauvais est proche de méchant: mauvais/méchant comme une gale (Grand Robert).

\section{2. moindre-plus petit}

Passons maintenant à l'autre opposition, celle de moindre - plus petit. Là, nous sommes en présence de proportions tout à fait différentes: seulement un bon tiers des exemples de notre corpus (53 ex.) présentent la forme synthétique, en face de 96 ex. de la forme analytique.

La règle générale selon laquelle le comparatif analytique est toujours réversible est confirmée:

(23) Alice ne perd jamais pied et elle est plus petite que moi (Jacqueline Michel, La déprime 26).

La forme synthétique, en revanche, peut être réversible ou non-réversible.

Quand elle est lexicalement non-réversible, il y a, comme nous venons de le voir pour pire, un certain nombre de bases lexicales qui se substituent à petit: la plus fréquente est faible, comme dans (24):

(24) On parlait de plusieurs nouveaux écrivains anglais (...). Je lus aussi un grand nombre de livres de guerre - de moindre qualité - spécialement imprimés aux U.S.A. pour les pays d'outre-mer (Simone de Beauvoir, La Force des Choses I 23),

ou, avec degré: 
(25) Sur un grand nombre de points, j'étais - Sartre aussi, quoique peut-être à un moindre degré - déplorablement abstraite (id., La Force de l'âge 191)

Dans d'autres cas, il s'agit plutôt de peu important:

(26) Cette révolution-là (...) s'accomplit sous nos yeux sans que personne proteste. Les assurances sociales ne constituent pas une révolution moindre puisqu'elles supposent que l'homme n'est pas libre ni responsable de son propre avenir. (Georges Simenon, Quand j'étais vieux I 83-84),

peu élevé:

(27) Un habitant de la ville paierait, pour ce logis, un loyer trois fois moindre (Tony Duvert, Journal d'un innocent 232),

ou simplement peu:

(28) Le peintre l'a-t-il interprété, lui aussi? -Moins que les deux autres figures, parce que, sans doute, il le trouvait d'un moindre intérêt dramatique (J.-L. Curtis, Thé 52-53).

Les cas de non-réversibilité lexicale mis à part, on est en face d'une vingtaine de cas de moindre comparatif, contre une centaine de cas de plus petit.

Beaucoup de linguistes ont eu recours, dans cette situation, à la notion concret-abstrait. C'est ce qu' ont fait, par exemple Hjelmslev 6 et Togeby ${ }^{7}$. La tradition française (et belge) se sert d'une distinction un peu différente en parlant d'une nuance morale (Wartburg-Zumthor §528; Grammaire Larousse; Le Bidois; Hanse).

La notion de concret-abstrait joue sans doute un rôle, mais il a été beaucoup exagéré, à mon avis. On peut donner raison à Togeby quand il dit qu'

(29) "On emploie exclusivement ceux-ci [plus petit ou moins grand] quand le sens est concret: La maison plus petite de ma tante Molly (Green, Terre 45)".

Dans des périodes antérieures de la langue, ce rôle a été plus important. Ainsi, au XVIIe siècle, on pouvait attribuer à des substantifs concrets, par moindre, une valeur abstraite :

(30) Je lis ce matin dans Sertorius: (...) Curieux emploi du mot "moindre": -De suivre les drapeaux d'un chef moindre que vous

6 op. cit.

7 Grammaire française I, §249. 
(Acte I, sc.1) - Ils étaient plus que rois; ils sont moindres qu'esclaves (Actes III, sc.1) (André Gide, Journal, 1943, cit. Grand Robert, moindre)

mais cette possibilité n'existe plus.

Pour le problème qui nous occupe, en tout cas, il serait plus fructueux de se tourner vers une autre distinction. Les cas de non-réversibilité mis à part, on se trouve en présence de substantifs qui sont d'un type particulier: des expressions de quantités ou de mesure:

amplitude, mesure, pourcentage, moyenne de vie, quantité, dépense, distance, écart (avec moindre);

délimitation, dimension, dose, part, dénominateur, taille (avec plus petit).

Là, nous retrouvons plutôt la distinction non-emphase avec les formes synthétiques - emphase avec les formes analytiques:

(31) le pourcentage des crimes est bien moindre à Mexico qu'à New York ou à Chicago (Beauvoir, Choses I 222)

(32) le licencié de 1968, qui vit objectivement dans des conditions bien plus favorables que son homologue de 1830, mesure cependant un bien moindre écart avec le niveau de vie des prolétaires contemporains (Jacques Perret, Inquiète Sorbonne 71)

face à

(33) Charles (...) est aussi fort que lui, plus râblé, quoiqu'il soit de plus petite taille (Paul Vialar, Le fusil à deux coups 93)

(34) un voile portant également une croix mais de plus petites dimensions (Sainte-Soline, Années 173).

\section{Superlatif}

\section{1. pire-plus mauvais}

Pour le superlatif de mauvais, la forme synthétique est également de loin la plus fréquente (90\% des cas; 415 ex. contre 44). Le plus grand nombre de ces cas présentent des exemples d'un superlatif lexicalement non-réversible. Nous rencontrons ici les mêmes catégories que pour le comparatif:

- intensif (où le correspondant au positif serait, plutôt que mauvais, grand): 
(35) et elle pense fortement qu'elle a eu affaire à l'un des pires salauds qui se puissent rencontrer (A.P. de Mandiargues, La Motocyclette 108),

ou, dans quelques cas, fort:

(36) Il entraînait ses hommes à supporter les pires chaleurs, sans boire ni manger (Lanoux, Mer 314-15);

- la base lexicale grave:

(37) La mère, les pommettes en feu, chuchotant des ragots venimeux dont le pire est qu'ils sont parfois vrais... (Françoise MalletJoris, Trois 144),

ou un autre adjectif négatif, parfois présent dans le contexte:

(38) Le vert est terrible, le froid vert est le pire (Paul Guimard, Les choses de la vie 148).

Dans certains cas, il s'agit d'une non-réversibilité totale (morphologique), ainsi parfois dans les emplois neutres: le pire:

(39) Les grands bourgeois de l'O.C.M. avaient au départ accepté le pire (Perrault, Traque 539),

où le pire équivaut plutôt à tout.

Cette non-réversibilité se rencontre surtout avec l'expression au pire (="au pire cas, au pis aller"):

(40) Au pire, ce ne serait pas la première fois qu'il aurait affronté une tornade (Saint Phalle, Souverain 194):

impossible de construire au positif *au mauvais.

En dehors de cette construction, l'emploi neutre de pire, comme au comparatif, représente le plus souvent une non-réversibilité lexicale:

(41) Attends...Tu ne connais pas le pire. Elle s'est jetée du clocher et s'est écrasée dans le cimetière. Le corps a été conduit à l'hôpital de Mantes. (Boileau-Narcejac, Sueurs froides 95),

(base lexicale: grave).

Dans la construction ce qui...de pire, il y a même non-réversibilité complète (morphologique et lexicale):

(42) Ce qui aurait pu arriver de pire, c'est qu'on nous en donnât (Gautier, Je 37)

(au positif: ce qui aurait pu arriver de mal).

Les exemples de pire superlatif non-réversible écartés, on reste en face d'une situation beaucoup plus équilibrée que devant les matériaux 
bruts: les exemples réversibles présentent 51 cas de pire, en face 44 de plus mauvais. Il faut alors de nouveau poser la question: quelle est la différence des deux superlatifs?

Si nous confrontons les exemples (43) et (44), avec un substantif noyau du type "temps":

(43) Il fallait toujours que dans les pires moments il arrive à Françoise de tomber sur un cave pareil (Alberte Sainte-Aube, Bienvenue à New York 147-48)

(44) Mais vous avez choisi la plus mauvaise semaine, Danielle (Hunebelle, Henry 217),

il apparaît que la forme analytique, dans (44) exprime une référence spécifique (au singulier), tandis que le syntagme nominal dans (43) exprime une référence non-spécifique (au pluriel).

Ainsi, la plupart des exemples avec pire/plus mauvais superlatif réversibles peuvent être ramenés à la distinction spécifique - non-spécifique, ainsi quand il s'agit du type de noyau "produit" ((45) et (46)):

(45) tu es persuadé que rien de vraiment mauvais ne peut sortir de toi, que ta pire toile, la plus bâclée, la plus dégueulasse a quand même sa petite lumière inimitable (Dutourd, Pluche 180)

(46) Mais croyez-moi, c'est sa plus mauvaise toile! (Guy des Cars, J'ose 226)

ou "humain" ((47) et (48)):

(47) -Tu sais, la plupart des filles n'osent rien dire chez elles. Ce sont les pires. Toi, tu es un copain et tu comprends... (Simenon, Les Innocents 78)

(48) Il se méfiait de cette équipe. C'était la plus mauvaise du Blossom (Robert Merle, L'île 13).

Seul un bon cinquième des exemples résistent à cette explication. Avec ces exemples (23, soit 5\% de notre corpus originel), on est donc contraint à trouver une autre explication:

(49) Le plus mauvais comédien peut avoir un fléchissement dans le détestable (Gautier, Je 264)

(50) Florence commanda un paquet de nouilles de la marque Butifer. "La pire", pensa Gaston qui n'en dit rien. (Kléber Haedens, Les Tilleuls 124),

qui pourrait être l'emphase ou l'expressivité. 


\section{2. moindre-plus petit}

Le superlatif de petit est dominé par la forme synthétique qui représente plus de $90 \%$ des cas (1165 ex., contre 109). Moindre, au superlatif, peut avoir une fonction comparative proprement dite, où il est réversible:

(51) Et ce salon que vous méprisez n'est qu'un petit abcès de cette décomposition. Et l'un des moindres, je vous l'assure (Sagan, Profil 145);

et en combinaison avec la négation:

(52) Ce rêve fut en quelque sorte le premier avertissement, et non le moindre (Carrière, Uzès 32),

mais ce cas, où il s'agit, comme on le voit, surtout de moindre substantivé, est très rare: il ne représente que $2 \%$ des exemples de moindre superlatif.

Le champ privilégié de moindre superlatif est avant tout un emploi généralisant (n'importe quel, tout, chaque):

(53) une rue, un escalier, une sonnette, le moindre décor, le moindre détail me dépaysait (Simone de Beauvoir, La Force de l'âge 370).

Avec négation (aucun):

(54) Martial aurait mis sa main à brûler que ni son fils ni son beaufrère n'avaient lu la moindre ligne des auteurs en question (Curtis, Roseau 32).

Dans ces cas, le superlatif est le plus souvent non-réversible. On peut distinguer plusieurs cas différents de cette non-réversibilité:

a) Elle peut être due au fait que le substantif noyau est une entité ingraduable, comme dans (55) et (56):

(55) mais quand le séjour à Bellerive ne devrait pas me rapporter le moindre dollar, j'irais tout de même là-bas (Marcel Aymé, Louisiane I 6)

(56) tous ces fonctionnaires qui ne savent pas ce que c'est que la richesse et que le moindre billet de 500F suffit à faire parler (Sarrazin, Crèche 76).

On n'imagine guère *un petit dollar ou *un petit billet de 500F: il n'y a pas de billets de dollar ou de 500F de différentes grandeurs. Si le superlatif apparaît, c'est ainsi uniquement pour apporter une nuance généralisante. 
b) Dans d'autres cas, la combinaison petit + noyau est aussi improbable, bien qu'il ne s'agisse pas d'une entité ingraduable:

(57) Il échouait constamment dans des endroits où il n'avait pas eu la moindre intention d'aller (Mallet-Joris, Allegra 74)

(58) Mme Nyha écoutait le vieux réciter ses litanies dans un langage rauque sans manifester la moindre impatience (Boulle, Oreilles $51)$.

c) Même si petit + substantif peut s'imaginer, il n'est pas toujours sûr que le superlatif soit réversible. Il arrive souvent que petit au positif donnerait un sens carrément faux, comme dans (59) et (60):

(59) ils finissaient par en connaître toutes les embûches, les roches tranchantes qui scient les pneus, les fondrières, les moindres racines (Boulle, Oreilles 29)

(60) Elle se conformait à l'image qu'on attendait d'elle, accédant aux moindres désirs de Mme Flahaut (Saint Phalle, Tournesol 168).

Il est possible de transposer ces superlatifs au positif en obtenant des phrases bien formées:

(59') (...)les roches tranchantes qui scient (...) les petites racines

(60') (...) accédant aux petits désirs de Mme Flahaut,

mais cette transposition donne un autre sens que celui des phrases primitives (59) et (60): le sens généralisant de "tout", "n'importe quel" disparaît, en laissant la place au sens plus précis de petit ("de petite taille"). Dans (59) et (60), le superlatif moindre, selon notre définition, est donc non-réversible: il peut certes être remplacé par le même adjectif (petit) au positif, mais avec changement de sens.

Je propose de rendre compte de ce changement de sens en ayant recours au terme de "focalisation" employé par Henning Nølke ${ }^{8}$. Dans (59') et (60'), petit est focalisé, c'est-à-dire que l'intérêt est concentré sur le contenu de cet adjectif. Dans (59) et (60), en revanche, il n'y a pas de focalisation.

d) Dans d'autres cas, le passage du superlatif au positif entraîne aussi une focalisation, mais d'un type particulier. Si l'on considère les exemples (61) et (62):

8 Linguistique modulaire, Louvain-Paris 1994; Det attributive adjektivs placering på fransk, i: Ny forskning i grammatik. Fællespublikation 3, Odense 1996, p. 49-83. 
(61) N'avait-il pas été en quelque sorte un bienfaiteur de la douloureuse humanité en égayant le moindre foyer? La société lui était redevable de ces objets d'art indestructibles. (Jean Cayrol, N'oubliez pas que nous nous aimons 55-56)

(62) puisqu'il ne me cachait rien, je me crus dispensée de me poser sur lui la moindre question (Beauvoir, La Force de l'âge 30),

on voit, en effet, que leur transposition au positif:

(61') N'avait-il pas été en quelque sorte un bienfaiteur de la douloureuse humanité en égayant un petit foyer?

(62') puisqu'il ne me cachait rien, je me crus dispensée de me poser sur lui une petite question

entraînerait un changement de référence: il s'agit, dans (61') et (62'), d'une référence spécifique, loin de la référence générique ("n'importe quel") de (61) et (62).

\section{Conclusion}

Dans ce qui précède, j'ai essayé de distinguer un emploi réversible des formes comparatives et un emploi non-réversible. Cette distinction a les avantages suivants:

a) en formulant la loi selon laquelle un comparatif et un superlatif analytique est toujours réversible, tandis qu'un comparatif ou un superlatif synthétique peut l'être, elle permet de rendre compte, dans une large mesure, de l'alternance comparaison synthétique - comparaison analytique;

b) elle permet, en écartant d'abord les cas de comparatif (superlatif) non-réversible, de montrer que - là où l'emploi du comparatif (superlatif) synthétique ou celui du comparatif (superlatif) analytique paraissait en majorité écrasante, les deux formes sont en réalité en équilibre;

c) du point de vue de la terminologie, elle rend superflue la notion de comparatif (superlatif) absolu;

d) du point de vue de la linguistique historique, elle offre un instrument plus précis pour décrire les changements diachroniques. J'espère revenir sur cette question dans un travail ultérieur. 